\section{Gefahr durch Gelatine-haltige Zäpfchen}

\begin{abstract}
apanische Forscher empfehlen, Sup$\mathcal{}$ positorien mit derselben Sorgfalt einzusetzen wie z. B. gelatinehaltige Vakzine. Nach ihren Ergebnissen ist die Zahl der sensibilisierten Kinder zwar niedrig, es ließ sich jedoch bei 10 Kindern ein Zusammenhang zwischen einer anaphylaktischen Reaktion und der Behandlung mit Gelatine-haltigen Suppositorien demonstrieren. Es handelte sich um Chloralhydrat-Zäpfchen, die zur Betäu-
\end{abstract}

bung gegeben worden waren. Der Gelatinegehalt eines Suppositoriums lag bei $231 \mathrm{mg}$. Fünf Kinder wiesen eine Anaphylaxie mit Hypotension oder Zyanose und auch Urtikaria oder pfeifende Atemgeräusche auf. Zwei weitere Patienten zeigten Pfeifgeräusche und Urtikaria, bei drei kam es nur zu Urtikaria.

red

Sakaguchi $\mathbf{M}$ et al. J Allergy Clin Immunol 2001; 108: 1033-4

\title{
Allergisch gegen Baudenkmäler
}

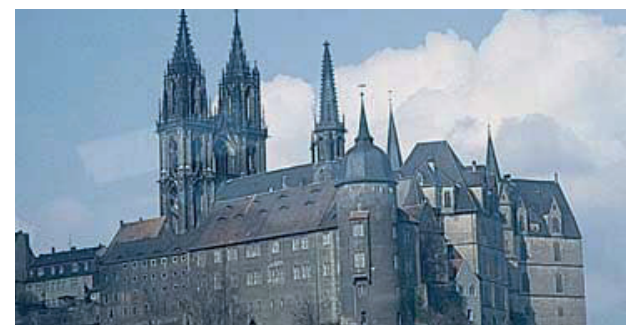

E ine Patientin mit bekannter ¿ Hühnerei-Allergie erlitt trotz einer Hühnerei-freien Diät nach einer 15monatigen symptomfreien Periode eine schwere Asthmaattacke. Der daraufhin festgestellte IgE-Antikörpertiter für Hühnerei lag bei mehr als $1000 \mathrm{kU} / \mathrm{l}$.
Die Herkunft der Allergene erwies sich als historisch: Die Patientin hatte bei ihrer Arbeit an der Restauration einer Kathedrale aus dem 16ten Jahrhundert Hühnerei-haltige Stäuben inhaliert.

Schon im alten Rom wurde dem Material zum Verputzen von Gebäuden Hühnerei beigemengt. Möglicherweise könnte diese über Jahrhunderte praktizierte Technik eine Rolle bei der Entwicklung eines berufsbedingten Asthmas bei Restauratoren spielen. $\quad f k$

Armentia A et al. N Engl J Med 2001; 345: 1068-9

\section{An juckenden Striemen war ein Speisepilz schuld}

$\mathrm{D}$ as allseits bekannte „Chinese-Restaurant-Syndrom" scheint Konkurrenz zu bekommen: Ein Toxin von Shiitake (weltweit der zweithäufigste gezüchtete Speisepilz) kann rote, striemenartige Hautreaktionen hervorrufen. Bislang ist diese Erkrankung vorwiegend in Japan beobachtet worden. N. Hass, Berlin, berichtete jedoch über einen 67-jährigen Patienten mit einer Shiitake-Dermatitis. Die Erkrankung ähnelt einer Urtikaria oder einer typischen Dermatose, die nach BleomycinGabe auftreten kann.

Nach intensiver Suche ermittelte Haas den Übeltäter - lokale Kortikosteroide und orale H2-Blocker machten den juckenden Striemen nach einigen Wo- chen ein Ende. Ein Grund, warum die Erkrankung in Japan so viel häufiger auftritt, könnte sein, dass dort die Pilze oft roh gegessen werden (das Toxin ist hitzelabil). „Vielleicht aber," so Haas, ,ist bisher nicht so darauf geachtet worden." uls

Haas N et al. Hautarzt 2001; 52: 132-5

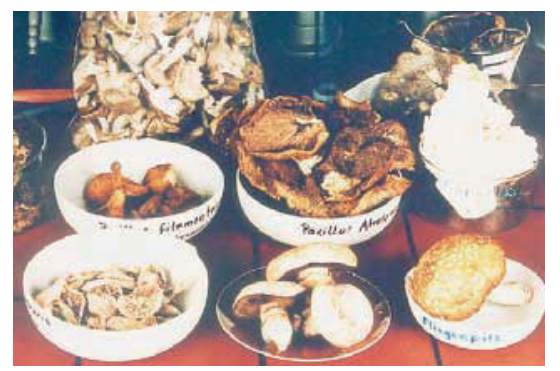

\section{A1-Gen lässt Mastzellen länger leben}

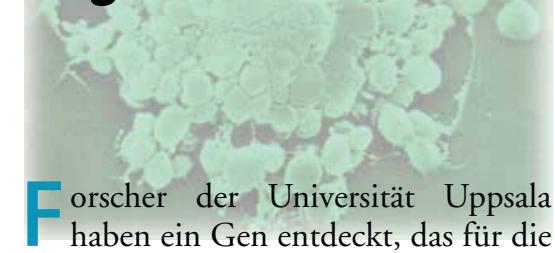
Persistenz der Mastzellen verantwortlich ist. Die Überlebensfähigkeit der Mastzellen führen die Forscher auf das Gen A1 zurück. A1 bewahrt Mastzellen vor dem programmierten Zelltod. Werden die Mastzellen durch IgE aktiviert, steigt die Expression des A1-Gens. Wird A1 blockiert, überleben die Mastzellen die allergische Reaktion nicht. Im Mäuseversuch zeigte sich, dass ein Mangel an A1-Genen auch insgesamt weniger Mastzellen nach einer allergischen Reaktion bedeutet. Für die Forscher tut sich damit möglicherweise ein neuer Behandlungsweg auf.

pte

Xiang Z et al. J Exp Med 2001; 194: 1561-70

\section{ADIZ 2002}

$\mathrm{D}$ as Bad Lippspringer Allergie-Dokumentations- und Informationszentrums (ADIZ) widmet sich neben der Arbeit mit Patienten der Weiterbildung des Fachpersonals aus Medizin, Pflege und Pharmazie. Zusätzlich zu den seit Jahren anerkannten Schulungen für Klinikärzte und Fortbildungen für Assistenz- und Pflegepersonal, die in Zusammenarbeit mit der Akademie für ärztliche Fortbildung in Münster aufgelegt wurden, konnten im Jahr 2001 auch strukturierte Weiterbildungsprogramme für Apotheker und deren pharmazeutisch-technisches Personal angeboten werden. Aufgrund der positiven Resonanz werden diese Veranstaltungen auch im Jahre 2002 weiterhin durchgeführt und themenspezifisch ausgebaut. Weitere Informationen über das Programm 2002 erhalten Sie bei: ADIZ, Burgstr. 12, D-33175 Bad Lippspringe, Tel. 05252 / 95-4500 oder -4502 , Internet: www.adiz.de 